\title{
Increasing Stability of Mine Surface Facilities on the Fill-Up Ground
}

\author{
Michail Sokolov ${ }^{1 *}$, and Sergey Prostov ${ }^{1}$ \\ ${ }^{1}$ T.F. Gorbachev Kuzbass State Technical University, 650000, 28 Vesennyaya St., Kemerovo, Russia
}

\begin{abstract}
The emergency condition of the mine surface facilities including a coal wash plant, a crusher, a bunker and three galleries, is considered. As a result of a complex of engineering-geological and geophysical surveys, it is established that the significant deformations of the foundations of the structure are caused by the zones of weakened (unconsolidated) soil in the bases of the foundations formed due to landslide developments in the bulk ground mass. The article describes the techniques for justifying the technological parameters of the consolidation grouting of soil bases including computer modeling, forecasting of deformations of the natural and consolidated ground mass, analysis of the stress-strain state of soils in the most dangerous area. The effectiveness of the adopted scheme of consolidation grouting of soil bases according to the approved methodology for elimination of the emergency condition of the mine surface facility was confirmed. The limit values of the calculated horizontal deformations in the soil bases of one of the supports of the galleries are determined. To prevent the indicated deformations, it was recommended to provide an additional row of injection wells with a depth below the border of the loosened soil zone.
\end{abstract}

\section{Introduction}

Stability of surface mine structures is an important condition of a safe technological process for the development and extraction of minerals. In some cases, in the foundations of such structures there are technogenic soils with different degrees of compaction and changeable deformation properties, which contribute to the development of uneven vertical displacement and are the causes of accidents. To improve the stability of the foundations of surface mine structures, methods of injection consolidation are used the most effective of which are methods of pressure injection [1,2] and electrochemical consolidation [3, 4]. The choice of approach to improving the stability of structures need to be supported by rock mechanics forecast, study of the stress-strain state of rock mass, including those with consolidating elements [5-7]. The use of software programs for geotechnical calculations using the finite element method significantly increases the efficiency, accuracy and speed of forecasting [8, 9].

\footnotetext{
*Corresponding author: ea-sokolov42@mail.ru
} 


\section{Materials and methods}

A seasonal coal wash plant with separators and galleries at Krasnobrodsky coal open-pit mine, the branch of OAO UK "Kuzbassrazrezugol" is an example of the above mine surface facilities. This facility includes a number of technological buildings and 3 galleries (Fig. 1). All structural elements have standard solutions, including post reinforced concrete foundations.

The facilities are located on the territory with a complex relief, formed as a result of mining operations and having a completely technogenic (man-made) character. On the site under consideration, the rock mass at a depth of 8 to $20 \mathrm{~m}$ is represented by man-made fillup soils in the form of a mixture of land waste, crushed stone, lumps, loam and sandy material compactly rolled. The fragments and fine aggregate are represented by sedimentary rocks of weathered sandstone type of low strength. Loam contains local lenses and nests of medium fine gravel with loamy hard, dense aggregate up to 35-45\%. In the hydrogeological respect, the site is characterized by the presence of an aquifer of groundwater in weathered and destroyed bedrock (formation-fractured waters).

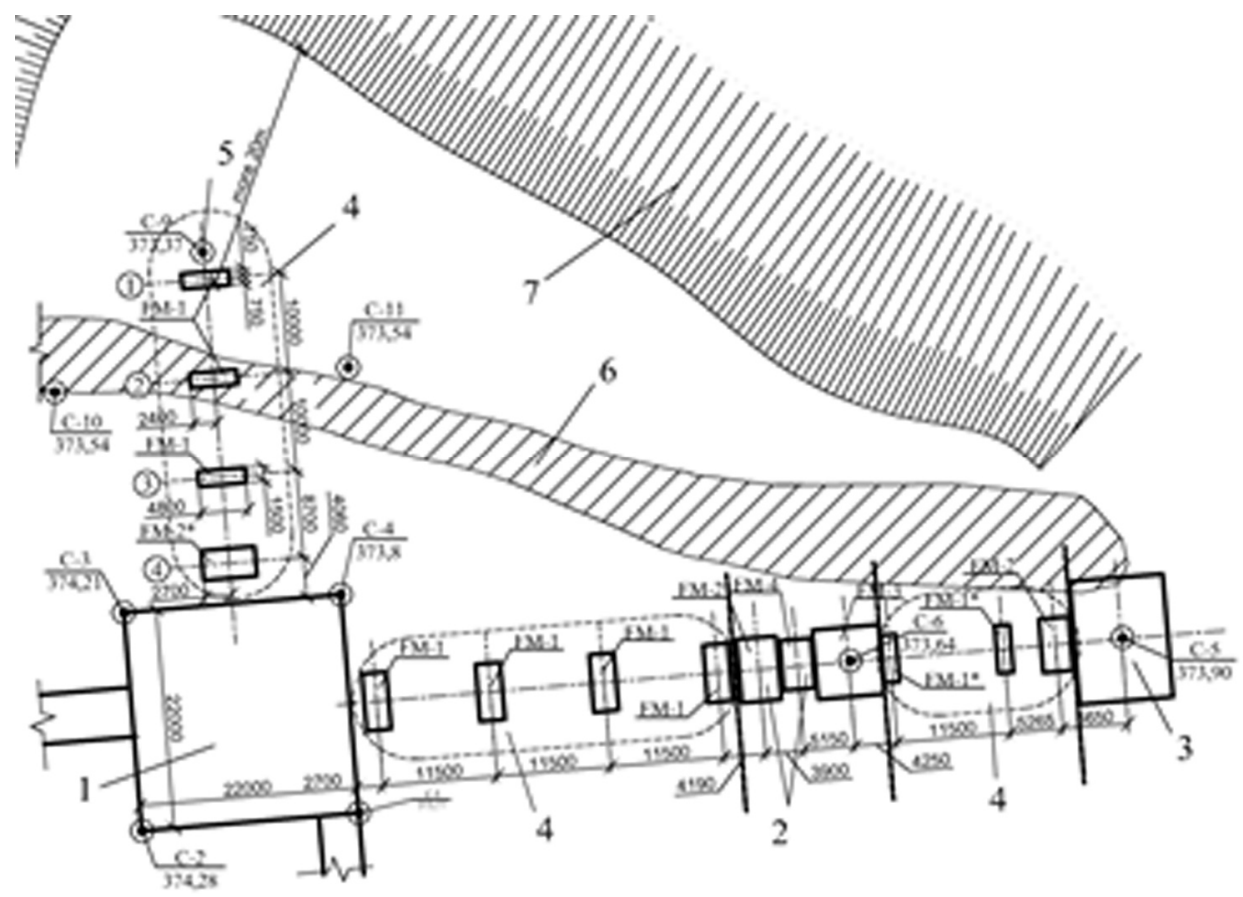

Fig. 1. Scheme of the territory and the plan of the investigated object: 1 - coal washing building; 2 crusher and crushing section; 3 - receiving hopper; 4 - galleries No. 11, No. 5, No. 9; 5 - geological wells; 6 - zone of soils decompaction; 7 - slope of fill-up foundation.

According to the data of visual surveys and engineering and geological surveys conducted by OOO NOOCENTR, the structure is in an emergency condition, it has significant uneven subsidence of the supports of the conveyor galleries. During the work on the surface of the site in the northeastern part, closed cracks of a landslide character (cleavage cracks) were found, and zones of decompaction were observed, which develop as a result of a partial displacement of the ground masses towards the unconsolidated slope of fill-up soil mass. Since a part of the receiving hopper and gallery No. 11 are located above the zones of decompaction, additional work has been carried out aimed at identifying and outlining these zones by geoelectric and radar control methods [10]. Analysis of the data of survey showed 
the need to perform measures to increase the load-bearing capacity of the soil base in the northeastern part by pressure injection methods, in particular under the foundations of the receiving hopper and supports of gallery No. 11, located directly above the zone of soil decompaction.

\section{Results and discussion}

The first stage of the analysis of the geomechanical state of the base of gallery No. 11 was aimed at predicting the vertical displacement of the surface along the longitudinal axis of the structures. The forecasting was carried out by mathematical modeling on the basis of the finite elements method and the modern geotechnical software package "Alterra" of OOO "InzhProektStroy", the Russian program developer. The obtained distribution graphs of the vertical displacement $\Delta h$ of the supports of gallery from the relative coordinate along the axis of the object $L_{i} / L_{\text {all }}$ (Fig. 2), where the total length $L_{\text {all }}=33.05 \mathrm{~m}$, show that the displacement has maximum values mainly at intervals located above the decompaction zones: in the ranges of $L_{i} / L_{\text {all }}=0.3 \ldots 0.4$ and $L_{i} / L_{\text {all }}=0.6 \ldots 0.7$.

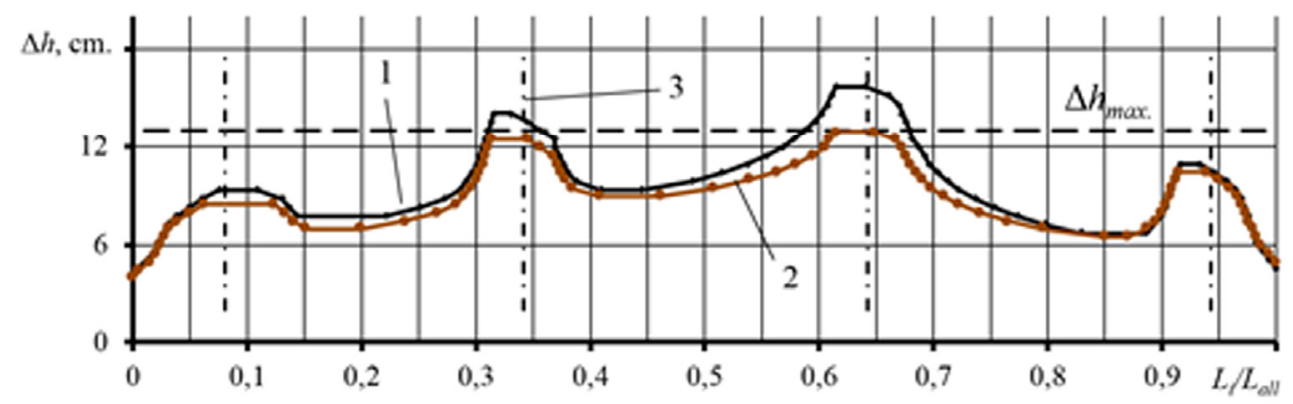

Fig. 2. Distribution of vertical displacement $\Delta h$ along the axis: 1 - with natural base; 2 - when consolidated according to the approved scheme; 3 - axes of foundations symmetry.

Taking into attention the maximum permissible size of subsidence of $\Delta h_{\max }=14 \mathrm{~cm}$, determined according to SP 23.13330.2011, it can be concluded that the proposed consolidation scheme allows us to achieve the required stability of the structure, however, the subsidence in the above ranges is close to the maximum permissible values, so it is advisable to conduct a more detailed study of individual supports of the gallery.

The second stage of modeling was aimed at studying and analyzing the stress-strain state of the base of potentially the most dangerous support of the gallery in the longitudinal and transverse directions. The schemes of basic models of the soil base of the gallery support are shown in Fig. 3.

The base was simulated from the top mark of the foundation with the specification of equivalent vertical pressure forces $P$, the horizontal load $T$ and the bending moment from deformation of the support in the transverse direction $M$. Foundations of FM-1 type are a slab with a width of $b_{f}=1.5 \mathrm{~m}$, a length of $l_{f}=4.8 \mathrm{~m}$ and a height of $h_{f}=0.6 \mathrm{~m}$, made of heavy monolithic reinforced concrete (density $\rho_{f}=2500 \mathrm{~kg} / \mathrm{m}^{3}$, elastic modulus $E_{f}=2000 \mathrm{MPa}$, Poisson's ratio $\left.v=0,15\right)$. The depth of foundation from the lowest mark of the surface was $d_{f}=3.090 \mathrm{~m}$. In the base of the foundation there is concrete preparation with a height $h=0.1 \mathrm{~m}$. The foundations are in open pits backfilled with large clastic technogenic deposits with a thickness of $0.8 \mathrm{~m}$. To solve the problem of the various kinds, the decompaction zones were averaged and divided into two identified characteristic regions. 
The physical and mechanical properties of the elements of the model are presented in the Table.

Based on the data given and the algorithms, described in the work [11], the main parameters of the model for the case under consideration were assigned: the width in the longitudinal direction $b_{m l}=41.0 \mathrm{~m}$, in the transverse direction $-b_{m 2}=31.0 \mathrm{~m}$ (taking into account the size of the pit and the foundation); height of the model is $h_{m}=21.0 \mathrm{~m}$. The boundary conditions in the form of restrictions of displacements are superimposed similarly to the models adopted in $[11,12]$. For the model in the longitudinal section, the load $P$ was set by two forces of magnitude $P / 2$ with a displacement of $l=0.6 \mathrm{~m}$ on either side of the side faces of the foundation, simulating the position of the support elements. In the crosssection, the loads $P, T$ and $M$ are given along the axis of symmetry of the foundation. In both cases, the upper edge of the foundation (cutoff) is considered the point of application of the load.
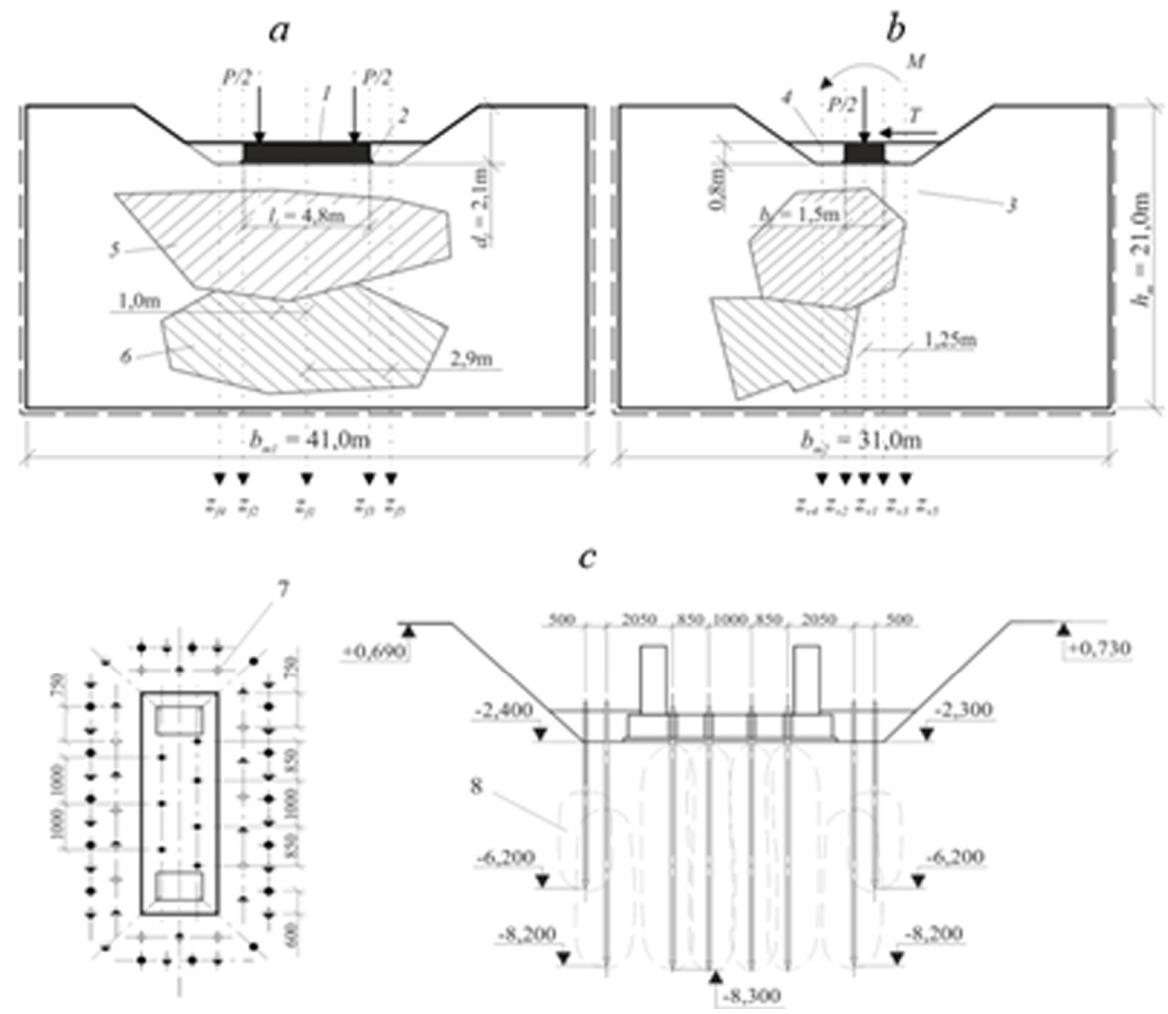

Fig. 3. Schemes of the basic model of support in the longitudinal $(a)$ and transverse $(b)$ directions and the location of consolidation zones $(c)$ : 1 - foundation; 2 - preparation; 3 - fill-up rock mass; 4 - technological backfilling; 5 - decompaction zone No. 1; 6 - decompaction zone No. 2; 7 - injector; 8 consolidation zone.

Table 1. Physico-mechanical properties of the elements of the model

\begin{tabular}{|c|c|c|c|c|c|c|}
\hline \# & $\begin{array}{c}\text { Name of the model ele- } \\
\text { ment }\end{array}$ & $\begin{array}{c}\begin{array}{c}\text { Density } \\
\text { of the } \\
\text { material }\end{array} \\
\text { (soil) } \rho, \\
\mathbf{k g} / \mathbf{m}^{3}\end{array}$ & $\begin{array}{c}\text { Modulus of } \\
\text { deformation } \\
E, \text { MPa }\end{array}$ & $\begin{array}{l}\text { Poisson's } \\
\text { ratio } v\end{array}$ & $\begin{array}{c}\text { Angle of } \\
\text { internal } \\
\text { friction } \\
\varphi, \text { deg. }\end{array}$ & $\begin{array}{c}\text { Bond } C, \\
\quad \mathrm{kPa}\end{array}$ \\
\hline 1 & $\begin{array}{l}\text { Reinforced concrete mat } \\
\text { foundation }\end{array}$ & 2500 & 2000 & 0.15 & - & - \\
\hline
\end{tabular}




\begin{tabular}{|c|c|c|c|c|c|c|}
\hline 2 & $\begin{array}{c}\text { Concrete preparation for } \\
\text { foundation }\end{array}$ & 2400 & 1800 & 0.15 & - & - \\
\hline 3 & $\begin{array}{c}\text { Backfilling of the } \\
\text { excavation }\end{array}$ & 1950 & 20.0 & 0.25 & 20.0 & - \\
\hline 4 & Soil bases & 2050 & 38.0 & 0.29 & 23.7 & 73.6 \\
\hline 5 & Decompaction zone No. 1 & 1900 & 17.0 & 0.36 & 25.0 & 25.0 \\
\hline 6 & Decompaction zone No. 2 & 1800 & 12.5 & 0.37 & 25.0 & 15.0 \\
\hline
\end{tabular}

The results of the second stage of modeling are reflected in the form of fields of isolines of the stress-strain state of the soil mass of a separate support in the longitudinal and transverse directions, shown in Fig. 4.

In the longitudinal direction the stress distribution due to the influence of the decompaction zones occurs asymmetrically with the presence of significant distortions at the boundaries of these zones. The zone of stress concentration remains in the area under the foundation, without significant displacements described in the work [12], and the introduction of the consolidation zones into the calculation considerably reduces the level of stresses and the area of their concentration. The influence of the consolidation zones can be traced in the form of redistribution of deformations from decompaction zone No. 1 to the decompaction zone No. 2 and the soil mass, the value of vertical displacement are greatly reducing. In the cross section, the stresses are distributed predominantly in the left support part of the rock mass due to the asymmetric load and the location of the decompaction zones in the rock mass relative to the foundation. Consolidation increases the asymmetry, loading mainly the zone of decompaction No. 2 (at a depth of $z_{2}=8 \mathrm{~m}$ ), but significantly reduces the horizontal stresses under the foundation, thereby preventing horizontal displacements. 


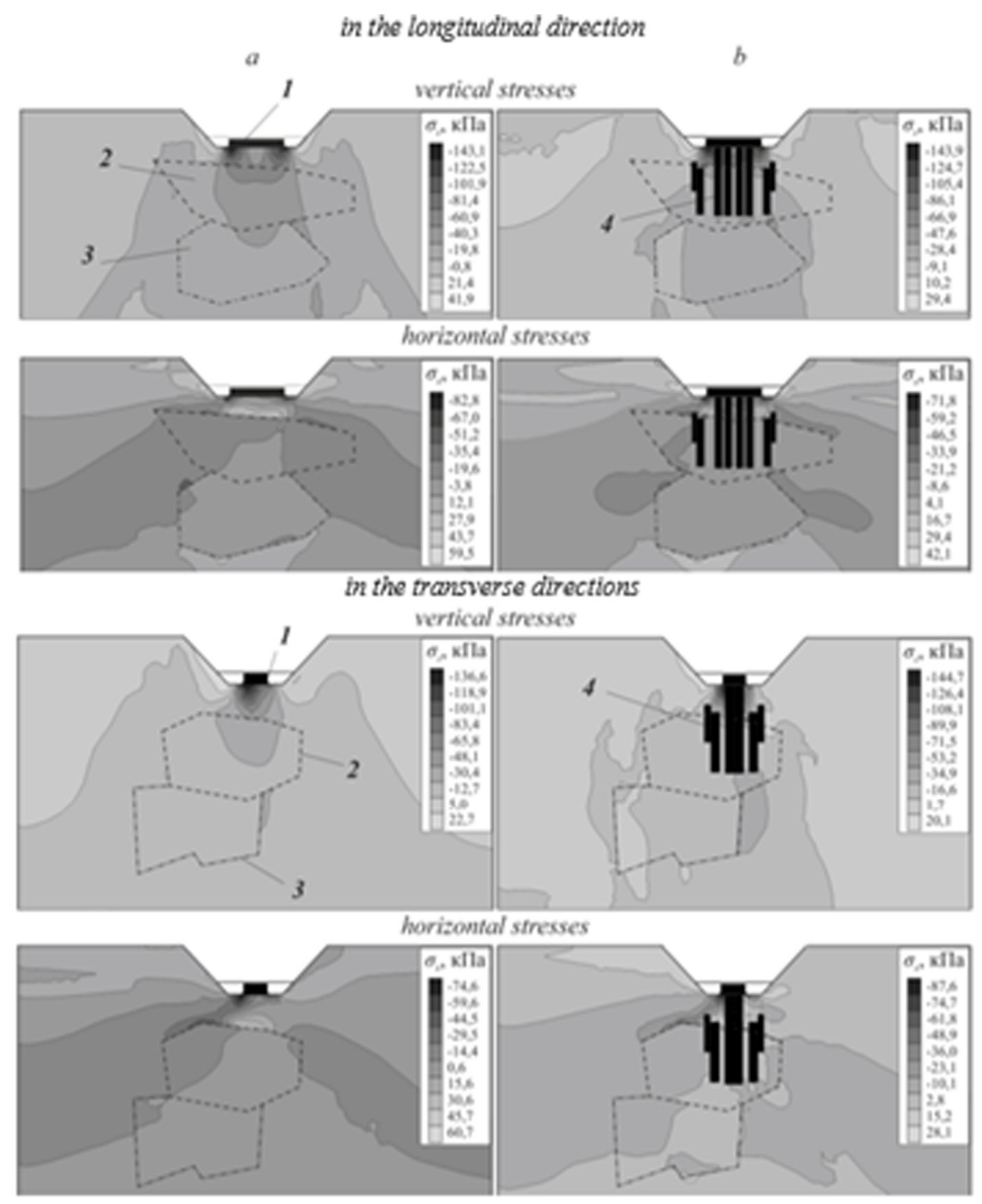

Fig. 4. Distribution of stresses in the natural and artificial soil mass in the longitudinal $(a)$ and transverse $(b)$ cross-sections: 1 - foundation; 2 - decompaction zone No. 1; 3 -zone of decompaction No. 2; 4 - consolidation zones.

The characteristic regularities in the distribution of vertical and horizontal deformations are shown in Fig. 5. The distribution of vertical deformations $\varepsilon_{z}$ has a similar character both in natural and in artificial soil mass. In the range of the near-surface part of the soil, the reduction of deformations is noted, and within the consolidation zones the deformation $\varepsilon_{z f}$ is much lower than in the natural soil mass; within decompaction zone No. 2 an increase in deformation $\varepsilon_{z f}$ is noted. Similar regularities can be traced in the cross section, however, additional concentrations of maximum deformations $\varepsilon_{z v}$ and redistribution of deformations into the soil mass are noted at a greater depth in the range of $z_{v i}=12.0 \ldots 17.0 \mathrm{~m}$. 
$a$
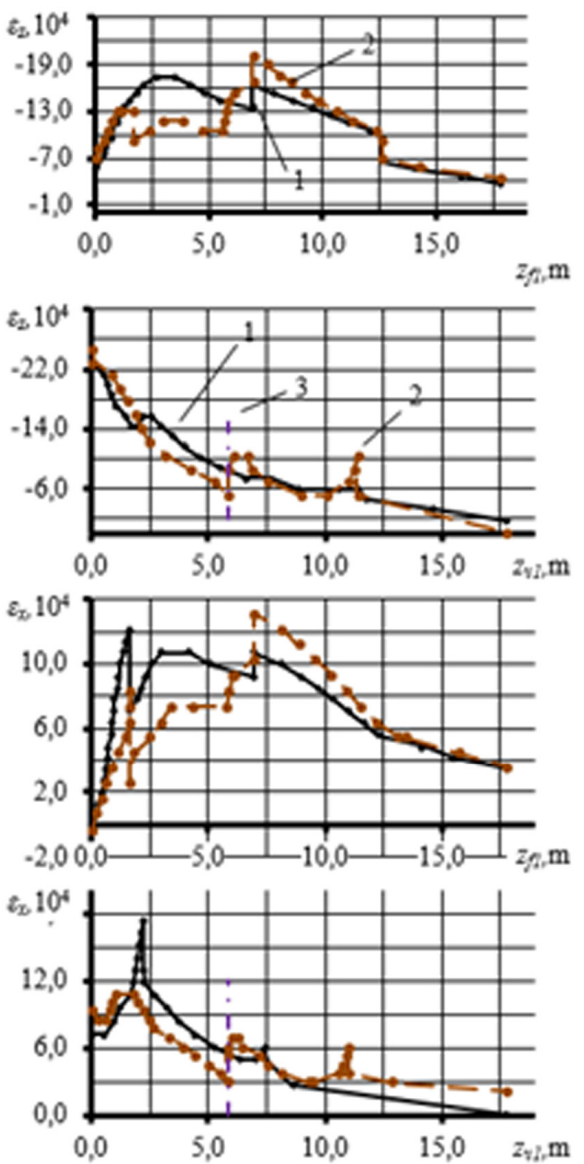

$b$
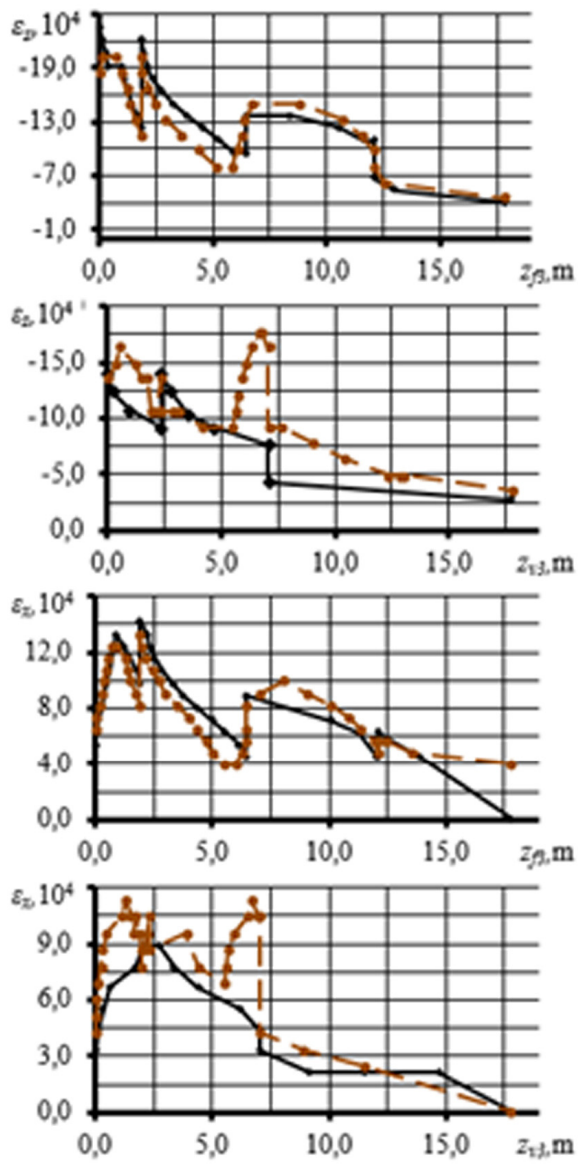

Fig. 5. Dependences of vertical $\varepsilon_{z}$ and horizontal $\varepsilon_{x}$ deformations at the depthьof model $z_{i}$ in longitudinal $(a)$ and transverse $(b)$ directions: 1 - natural soil; 2 - consolidated soil; 3 - boundaries of the consolidation zone.

The distribution of horizontal deformations $\varepsilon_{x}$ in both sections occurs mainly monotonically with the formation of separate local "bursts," mainly at the borders with decompaction zones. In the longitudinal section, there is a slight decrease in deformations $\varepsilon_{x f}$ in the artificial soil mass, as well as their noticeable increase in the regions below the consolidation zones. When calculating the artificial soil base it should be taken into account that in the longitudinal direction the decompression zone is subject to considerable horizontal deformation. In the transverse profile there are similar patterns, at the same time, the following anomalies are recorded: the formation of an additional deformation difference $\varepsilon_{x v}$ at $z_{v l}>11.3 \mathrm{~m}$; change in the nature of the distribution and increase in the values of deformations $\varepsilon_{x v}$ in the interval $z_{v 3}=0.0 \ldots 7.2 \mathrm{~m}$; local increase in deformation within the consolidation zone. Thus, the consolidation zones located in the direction of the action of the horizontal load can have significant transverse deformations.

\section{Conclusions}

Geomechanical analysis has proved the effectiveness of the method of injection grouting in managing the properties of weak soils and the stability of structures. Methods of mathemat- 
ical and computer modeling allowed us to predict with sufficient detail the effect obtained from consolidation and to identify the most problematic areas of the soil. In particular, in the presence of additional horizontal load and decompaction zones it is necessary to increase the number and depth of injection wells to prevent increased vertical and horizontal deformations. Based on the modeling data, the effectiveness of the consolidation scheme developed by OOO NOOCENTR has been proved, however, in order to achieve the greatest effect, it is recommended to place additional consolidation zones in the most loaded part of the soil mass at an asymmetric load, immersed in the ground to the depth of decompaction zone No. 2. Such an approach will allow us to redistribute vertical and horizontal stresses to a stronger soil mass and reduce the foundation subsidence, its tilt displacement and transverse deformations of the consolidation zones themselves.

\section{References}

1. M. N. Ibragimov, Rock Mechanics and Foundation Engineering, 50:5, 200 (2013)

2. M. N. Ibragimov, Rock Mechanics and Foundation Engineering, 52:2, 100 (2015)

3. Shao-Chi Chien, Fu-Chen Teng, Chang-Yu Ou, Acta Geotechnica, 10:6, 813 (2015)

4. A. Flora, S. Gargano, S. Lirer, L. Mele, Geotechnical and Geological Engineering. (2017)

5. A. V. Azarov, M. V. Kurlenya, A. V. Patutin, S. V. Serdyukov, Journal of Mining Science, 51:6, 1063 (2015)

6. T. V. Lobanova, S. V. Moiseev, Journal of Mining Science, 45:3, 227 (2009)

7. S. G. Bezvolev, Soil Mechanics and Foundation Engineering, 45:3, 77 (2008)

8. Z. G. Ter-Martirosyan, A. S. Abdulmalek, Soil Mechanics and Foundation Engineering, 44:6, 195 (2007)

9. D. M. Shapiro, N. N. Mel'nichuk, Soil Mechanics and Foundation Engineering, 44:2, $62(2007)$

10. S. M. Prostov, N. Yu. Nikulin, Journal of Mining Science, 51:5, 908 (2015)

11. S. Prostov, V. Sokolov, Taishan Academic Forum - Project on Mine Disaster Prevention and Control, 350 (2014)

12. M. Prostov, V. Sokolov, V. Pokatilov, International Journal of Applied Engineering Research, 10, 45297 (2015) 